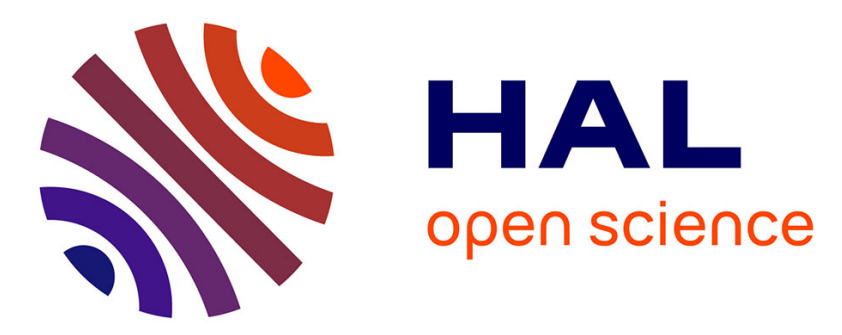

\title{
Impact of particles size and multiple scattering on the propagation of waves in stealth hyperuniform media
}

Adrien Rohfritsch, Jean-Marc Conoir, Tony Valier-Brasier, Régis Marchiano

\section{To cite this version:}

Adrien Rohfritsch, Jean-Marc Conoir, Tony Valier-Brasier, Régis Marchiano. Impact of particles size and multiple scattering on the propagation of waves in stealth hyperuniform media. Physical Review E , 2020, 10.1103/PhysRevE.102.053001 . hal-02896698v4

\section{HAL Id: hal-02896698 \\ https://hal.science/hal-02896698v4}

Submitted on 26 May 2021

HAL is a multi-disciplinary open access archive for the deposit and dissemination of scientific research documents, whether they are published or not. The documents may come from teaching and research institutions in France or abroad, or from public or private research centers.
L'archive ouverte pluridisciplinaire HAL, est destinée au dépôt et à la diffusion de documents scientifiques de niveau recherche, publiés ou non, émanant des établissements d'enseignement et de recherche français ou étrangers, des laboratoires publics ou privés. 


\title{
Impact of particles size and multiple scattering on the propagation of waves in stealthy-hyperuniform media
}

\author{
Adrien Rohfritsch, Jean-Marc Conoir, Tony Valier-Brasier, and Régis Marchiano \\ Sorbonne Université, CNRS, Institut Jean Le Rond d'Alembert, \\ UMR 7190, 4 Place Jussieu, Paris, F-75005, France
}

(Dated: May 26, 2021)

\begin{abstract}
Propagation of waves in materials that exhibit stealthy-hyperuniform long range correlations is investigated. By using a modal decomposition of the field that takes multiple scattering into account at all orders, we study the impact of the concentration of particles on the transparency of such materials at low frequency. An upper frequency limit for transparency is defined that include both the particles size and the degree of stealthiness. We show that the independent scattering approximation is not relevant to calculate elastic mean free paths when wavelength become comparable to the size of particles. We find that transparency is very robust with regard to the degree of heterogeneity of the host random medium and the polydispersity of particles. Finally, it is shown that resonances can be used as frequency filter.
\end{abstract}

\section{INTRODUCTION}

The study of wave propagation in random heterogeneous media has been in constant evolution from the last century. Understanding and predicting the propagation of waves while multiple scattering occurs is a challenge that is in constant evolution. Multiple scattering in random media is of practical interest in many fields of physics ranging from the theory of randomly disordered crystals [1], the resonant scattering of light [2], the ultrasonic monitoring of colloidal mixtures [3], the effective dynamic mass density of composites [4], metamaterials [5] to name a few.

Reviews of the best-known models and their mathematical backgrounds can be found in Refs. [6-9]. Many of these models, at their best, are based on the use of paircorrelation functions in order to describe the microstructure of the random media and the Percus-Yevick approximation to take account the correlation between particles [10-12]. By nature, they are limited to describe media which are statistically uniform. An evolution has been noticeable at the turn of the two past decades where the tuning of the microstructure has been widely explored to design materials with specific properties, as the cloaking for example [13].

Recently, a new state of matter has been discovered that is characterized by cancellation of normalized density fluctuations at infinite wavelength. This state is called hyperuniformity (or superhomogeneity) [14, 15] and lead to very particular and interesting properties that have already been observed at natural mesoscopic [16] or microscopic scales [17]. Self assembly of dense packings can also be hyperuniform $[18,19]$. Among hyperuniform media, a subclass exist for which normalized density fluctuations cancel for finite wavelengths $\lambda>\lambda_{\min }$. These ones are called stealthy hyperuniform (SHU) and are at the center of our study. In this context, several recent works have shown the importance of employing $n$-points correlation functions of order higher than two for the effective dielectric tensor of electromag- netic waves propagating in a two-phase composite random medium with isotropic components [20, 21].

Drawing on this experience, it seemed interesting to use a numerical software to calculate the wave propagation through a disordered distribution of particles because there is a priori no consideration on the correlation in such approach. By correlation, we understand not only the geometric arrangement of the particles but also the constructive or destructive interferences between the waves which propagate in the medium. In the following, we will use an in-house software, called MuScat, which is able to address this problem with several tens of thousand cylinders, regardless of the type of cylinders with high contrast ratio [22] or polydispersion, the frequency range investigated, and the kind of geometry [23]. This allows us to model any microstructure made of randomly distributed cylinders in detail without approximation except the inherent numerical errors.

To illustrate the great potential of stealth hyperuniformity, one can cite the numerous studies dealing with the emergence of band gaps in SHU materials that have a bandwidth comparable to periodic structures, but are isotropic $[24,25]$. A recent remarkable numerical study has investigated this phenomenon for elastic waves propagating in a medium with rather large particles [28]. The band gap effect typically appears for wavelengths $\lambda<\lambda_{\min }$. Combining advantages of ordered and disordered media, it allows interesting applications such as freeform waveguiding $[27,28]$ or optical cavities [29]. These phenomena - although very interesting - are out of the scope of our study. For $\lambda>\lambda_{\min }$, the cancellation of normalized density fluctuations induces transparency of the medium. However, this property is derived from a point pattern analysis, the question of the impact of the size of the scatterers being still open. Furthermore, strong multiple scattering effects are also neglected in general, considering that only weak interactions occur between the particles of the medium. A recent remarkable study by Kim and Torquato has already shown that wavelength can be finite compared to particles size without losing transparency [21]. In order to analyze this 
topic, they introduce an attenuation function that incorporates the contributions from all diffracted waves due to single (elastic) scattering events. Furthermore, Leseur et al. have proposed a transparency criterion for pointscatterers (monopoles) in the multiple scattering regime that depends both on the size $L$ of the medium considered and on the scattering mean free path of an uncorrelated medium [30]. This theoretical result has been recently qualified by an experimental study [26].

Following on from these recent works, we tackle here at the same time the two questions of strong multiple scattering and particles size effects on transparency. To do that, we focus on the propagation of acoustic waves in two dimensional SHU media made of elastic cylinders with large radius. In this case, cylinders can no longer be considered as point-scatterers, and the influence of the multiple scattering increases with the size of radii so that interactions between particles become more complicated and might go beyond single scattering events. The software MuScat allows exactly to face this topic (see Appendix to have details on the modeling). In addition, it allows us to introduce an important acoustic parameter for realistic clustering distribution which is the concentration of particles, denoted $\phi$. The position of the particles in SHU is usually controlled by the degree of stealthiness $\chi[31]$. Once the position of particles/cylinders is fixed, thanks to $\chi$, the concentration $\phi$ can be increased by growing the size of the cylinders. This is what we do in the following. This leads to characterize SHU by the parameters $\chi$ and $\phi$.

\section{SHORT AND LONG RANGE CORRELATIONS IN MEDIA WITH LARGE PARTICLES}

\section{A. Short range correlations}

In heterogeneous media constituted by finite size cylinders, a minimal exclusion distance $b=2 a$ between their centers is naturally imposed by their non-penetrability. This distance can also be larger but has to be lower than a maximum value imposed by the concentration. For random monodisperse media, the maximum concentration being $\phi_{r} \approx 83 \%$, the upper limit for $b$ is [22]

$$
b_{\max }=2 a \sqrt{\frac{\phi_{r}}{\phi}} .
$$

This type of constraints is called "short-range correlations" (SRC) because the presence of a cylinder only constraints its neighbourhood, that is to say the position of cylinders located in a circle of radius $b$ around it. For all systems considered in this paper, the minimal exclusion distance $b=2 a$ has to be taken into account in order to avoid overlapping. Imposing $b \geq 2 a$ strongly and variously affect propagation of waves, both at large and small wavelength regimes [22]. Important effects around resonant frequencies can also appear and were recently studied in Refs. [22, 32]. Interestingly, SRC media can be seen as an intermediate state between purely random and SHU media, as illustrated in Figure 1. Practically, to build SRC medium, we use the method known as Random Sequential Addition Process.

\section{B. Long range correlations}

The generation of SHU media is performed following an adaptation of the procedure described by Uche et al. [33]. The main quantities are shortly recalled here.

The point pattern representing the centers of the particles can be described either in real space by the pair correlation function $g_{2}(\mathbf{r})$ or in Fourier space by the structure factor $S(\mathbf{k})$ given for a pattern of $N$ points located at $\left(\mathbf{r}_{1}, \mathbf{r}_{2}, \ldots, \mathbf{r}_{N}\right)$ by

$$
S(\mathbf{k})=\frac{1}{N}\left|\sum_{j=1}^{N} e^{i \mathbf{k} \cdot \mathbf{r}_{\mathbf{j}}}\right|^{2}
$$

A point pattern is stealthy-hyperuniform if its structure factor $S(\mathbf{k})$ vanishes around the origin

$$
S(|\mathbf{k}|<K)=0 .
$$

Generally speaking, the bound $K>0$ can depend on the orientation of the vector $\mathbf{k}$, which means that the region where the structure factor vanishes can be arbitrary. Choosing a square domain $\Omega$ of side $K$ is convenient to get an analytical expression of $S(|\mathbf{k}|<K)$. $K$ is then given as a function of the number of particles $N$, the size of the medium $L$ and the degree of stealthiness $\chi$ as [30]

$$
K=\frac{\pi}{L} \sqrt{4 \chi N+1}
$$

The parameter $\chi$ is the ratio between the number $M(\Omega)$ of independent vectors in the domain $\Omega$ in reciprocal space and the number of degrees of freedom in real space ( $2 N$ in two dimensional space) [33]

$$
\chi=\frac{M(\Omega)}{2 N} .
$$

Extreme values $\chi \rightarrow 0$ and $\chi \rightarrow 1$ lead respectively to Poisson distribution and perfect crystal lattice. Both parameters $M(\Omega)$ and $N$ are finite because only finite systems can be considered numerically. The apodization of the infinite medium by a rectangular window (in both space directions) leads to the discretization of the reciprocal space. The spectrum is hence descretized in $P=\left|\frac{2 K}{2 \pi / L}\right|$ points. This apodization also imposes that $S(\mathbf{k}=\mathbf{0})=N$ rather than 0 . For a square domain $\Omega$ of size $2 K$, the structure factor can be written analytically in a potential form which can be minimized in order to 
reach stealth hyperuniformity $[30,33]$. A recent work focuses on other very efficient strategy to build very large hyperuniform packings [34].

In a wave scattering context, the structure factor appears in the expression of the scattered intensity as $S(\mathbf{q})=S\left(\mathbf{k}_{\mathbf{i}}-\mathbf{k}_{\mathbf{s}}\right)$, where $\mathbf{k}_{\mathbf{i}}$ is the incident wavenumber and $\mathbf{k}_{\mathbf{s}}$ the scattered wavenumber. Noting $k=\left\|\mathbf{k}_{\mathbf{i}}\right\|=$ $\left\|\mathbf{k}_{\mathbf{s}}\right\|$, this leads to the upper limit $k<K / 2$ for which, under monopolar and independent scattering approximations, only forward scattering appears which is called "perfect transmission" or "transparency" effect. For the sake of legibility, we introduce the reduce wavenumber $\tilde{k}=2 k / K$.

The word "transparency" has here to be understood as a propagation without loss (effective attenuation equal 0) rather than no perturbation of the total field in transmission [21]. Interesting properties such as complete photonic/phononic band gaps in SHU media with circular stealthy regions are known to be isotropic up to $\chi \approx 0.57$ $[31,35]$. For higher values, anistropric effects appear for wavenumbers $\tilde{k}>1$.

The main objective of this work is to tackle the question of the conservation of transparency in dense material with large particles. As already mentioned, it is worth noting that both short and long range correlations have to be imposed in the same time, due to the non-interpenetrability of the particles.

For each case presented in this paper, except in Section VI, particles are infinite steel cylinders with properties $c_{L}=5700 \mathrm{~m} / \mathrm{s}, c_{T}=3000 \mathrm{~m} / \mathrm{s}, \rho=7850 \mathrm{~kg} / \mathrm{m}^{3}$. The host medium is water $\left(c_{0}=1500 \mathrm{~m} / \mathrm{s}\right.$ and $\rho=1000$ $\mathrm{kg} / \mathrm{m}^{3}$ ) with wavenumber $k=\omega / c_{0}$. These properties are interesting because on one hand they lead to a strong impedance variation between particles and host medium (which is the case in many situations in wave physics) and on the other hand it allows a quantitative comparison with previous work that deals with SRC [22]. The source is a plane wave propagating in $x$-direction $\left(p_{\text {inc }}(\mathbf{r})=A e^{i k x}=A e^{i k r \cos (\theta)}\right)$. Next section is dedicated to the derivation of an expression for the theoretical upper limit for transparency in SHU media.

\section{Upper theoretical $a / \lambda$ limit for transparency}

Before going further, it is worth pointing out that it is impossible to build SHU materials transparent for arbitrary high ratio $a / \lambda$. To explain that, let us recall that the maximum concentration in $2 \mathrm{D}$ periodic distribution is $\phi_{p}=\pi /(2 \sqrt{3}) \approx 90.7 \%$ and approximately $\phi_{r} \approx 83 \%$ for random packings. Having that in mind, let us consider an arbitrary surface of size $L \times L$ with concentration $\phi$, noting $N=\phi L^{2} / \pi a^{2}$ the number of particles and $a$ the radius of the particles. As said in Section II, SHU materials are transparent for $\tilde{k}<1, K$ being given by Eq. (4). It gives in the limit $4 \chi N \gg 1$, that

$$
k a<\frac{K a}{2}=\sqrt{\pi \phi \chi} \leq \sqrt{\pi \phi_{p} \chi} .
$$

Eq. (6) shows that it is impossible to arbitrarily enlarge the domain $\Omega$ when considering media composed of finite-size particles. Secondly, it imposed an upper limit $a / \lambda$ for transparency that is reachable for a given concentration $\phi$. The limit case $\chi=1$ and $\phi=\phi_{p}$ leads to $(k a)_{\max }=1.68$, which leads to $\lambda \approx 3.74 a$. In this situation, both long range and short range correlations are maximized and the medium is a perfect crystal. This extreme situation is not reached in the following. We chose to impose at most $\chi_{\max }=0.6$ and $\phi_{\max }=50 \%$, which gives $(k a)_{\max }=0.97(\lambda=6.47 a)$, which already corresponds to large particles, and which is an "intermediate" frequency regime very common in acoustics.

It is worth noting that Eq. (6) mixes together the concentration $\phi$, related to SRC for large particles, and the parameter $\chi$ which is linked with long range correlation. As long as parameter $\chi$ is only a function of the $N$ points coordinates, for low values (typically $\chi<0.4$ ), two points can be very close to one another. This leads to the idea already known [36] that for a given particles size (and thus a given concentration), a minimum value of parameter $\chi$ is imposed. This explains why $\chi$ stays relatively high in this work $\left(\chi_{\min }=0.4\right)$.

\section{TRANSMISSION IN SHU MEDIA WITH FINITE-SIZE PARTICLES}

This section is dedicated to the study of the transmission through dense media with large particles at low and high concentrations.

\section{A. Transmission in systems with large particles at low concentration}

In real space, SHU media are not easily recognizable. In Figure 1, three distributions are shown : SRC, $b / 2 a=$ 1 (a), SRC, $b / 2 a=2.24$ (b) and SHU (c), with their structure factors. Properties of SRC are not enough to reach stealth hyperuniformity since the structure factor does not fully vanishes around the origin.

An illustration of perfect transmission is given in Figure 2 , representing the energy field scattered by a SHU medium composed of 6400 steel rods immersed in water, for two different wavenumbers : in the case $\tilde{k}=$ 0.929 , only forward scattering is observed, whereas for $\tilde{k}=1.047$, the backscattering effects become stronger. These two frequencies have been chosen close to $\tilde{k}=1$ to show that the transition appears to be very sharp. These two fields being calculated over only one distribution, no averaging over disorder is performed. This illustrates the surprising impact of correlations, leading to results very 
(a)

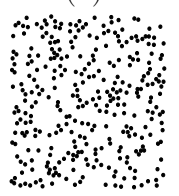

(d)

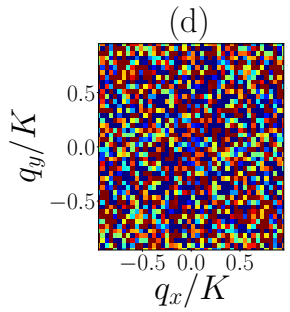

(b)

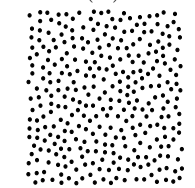

(e)

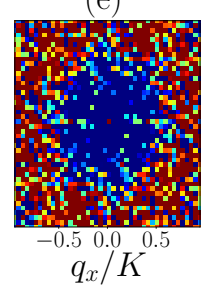

(c)
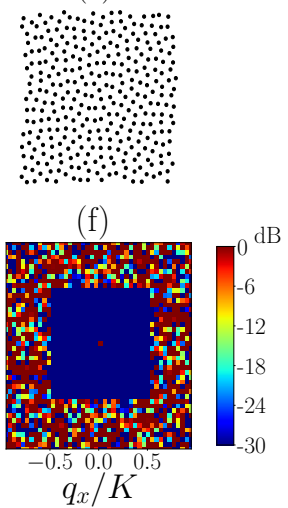

FIG. 1. (Color online) Random packings of cylinders (SRC with $b / 2 a=1)(\mathrm{a})$, short-range correlated medium $(b / 2 a=$ $\sqrt{0.5 / \phi}=2.24)(\mathrm{b})$ and stealthy hyperuniform medium (c) $(\chi=0.4)$ with their structure factors (respectively $(\mathrm{d}),(\mathrm{e})$ and (f)).
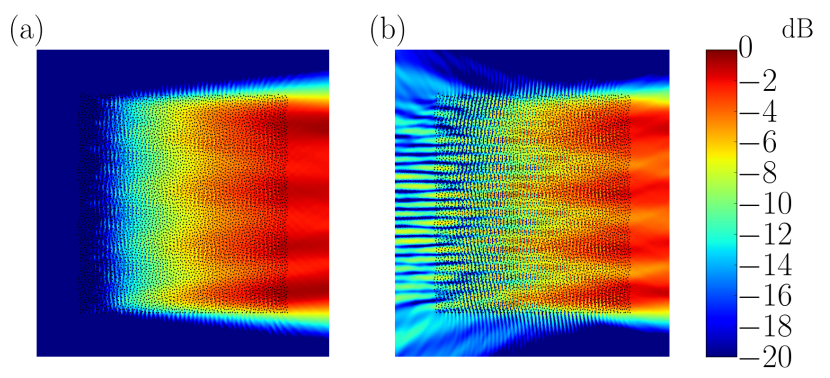

FIG. 2. (Color online) Scattered energy field with a incidence plane wave propagating from the left to the right for $\tilde{k}=0.929$ (a) and $\tilde{k}=1.047$ (b), for distributions made of $N=6400$ particles of radius $a=5 \mathrm{~mm}$, with $\chi=0.4$, with concentration $\phi=3.1 \%$ and $N / L^{2}=400$.

different from pure random distributions for which the incoherent part of the field can be much stronger.

In order to illustrate further the effect of hyperuniformity, reflection/transmission coefficients $R / T$ are defined such as

$$
\left\{\begin{array}{l}
R=\frac{\left|p_{R}\right|^{2}}{\left|p_{R}\right|^{2}+\left|p_{T}\right|^{2}}, \\
T=\frac{\left|p_{T}\right|^{2}}{\left|p_{R}\right|^{2}+\left|p_{T}\right|^{2}},
\end{array}\right.
$$

with $p_{R}$ and $p_{T}$ the scattered acoustic field at positions $x=-L / 2$ and $x=L / 2$, averaged on the transverse direction $y$. The source being a plane wave, the average process is only performed over $|y|<0.2 L$ in order to avoid diffraction effects. Expressions of Eq. (7) are chosen because they impose in a trivial way $R+T=1$.

In the single scattering regime, the transmission coefficient $T$ through a slab can be totally determined with the help of the structure factor. $S(\mathbf{q}) \rightarrow 0$ implies $T \rightarrow 1$, and at the opposite $S(\mathbf{q}) \gg 0$ implies an increase of the

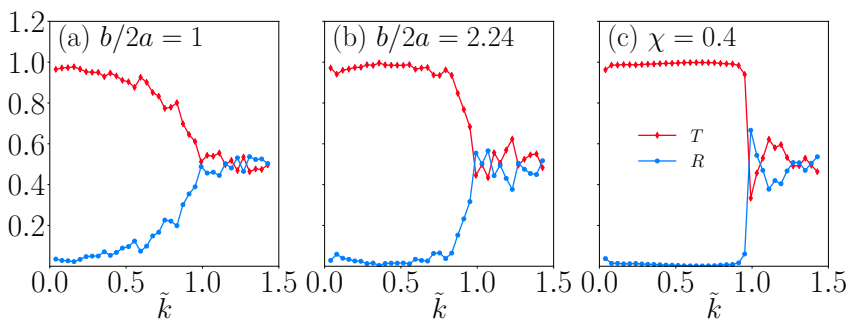

FIG. 3. (Color online) Reflection and transmission coefficients through short-range correlated medium with $b / 2 a=1$ (a), $\mathrm{b} / 2 \mathrm{a}=2.24$ (b) and stealthy-hyperuniform (c). All distributions are built with $N / L^{2}=1270$.

coefficient $R$. To illustrate that in several configurations of disorder, $R$ and $T$ coefficients for $b / 2 a=1, b / 2 a=2.24$ and SHU distributions are shown in Figure 3 (same types of distributions than the ones presented in Figure 1). The more the structure factor is close to 0 , the more $T$ gets close to 1 . Here, the particles radii are $a=5 \mathrm{~mm}$ and the concentration is $\phi=10 \%$, leading to the upper limit for transparency $K a / 2=0.35$. This is a regime for which the monopolar mode is already insufficient: $N_{m}=3$ is necessary to reach convergence (for elastic particles, scattering modes $T_{0}$ and $T_{1}$ are of same amplitudes for large wavelength, $T_{1}$ can never be neglected).

Before continuing, let us look at both Figures 1 and 3 together. It is interesting to note that SRC materials appear as a transition between purely random and SHU materials. This suggests another reading of the SRC materials. In fact, the local constraint imposed by the exclusion radius $b$ forces the medium to organize itself over a long distance as soon as the concentration is blocked. We then can understand why their behaviors tend to behave like those of SHU materials, at least at low frequency, even if they stay of course very different.

The perfect transmission property of SHU materials with non-ponctual particles has been demonstrated in the case of low concentrations. The comparison between other types of correlation $(b / 2 a=1$ and $b / 2 a>1)$ have illustrated the specific behaviour of SHU materials.

\section{B. Transmission in systems with large particles at high concentration}

In this section, the question of the reflection/transmission ratio in SHU media is discussed through the evolution of parameter $T$ with regard to the couple $(a, L)$. To this end, it is interesting to make an average of $\mathrm{T}$, either on $N_{\text {config }}$ realisations of the disorder $\langle T\rangle=N_{\text {config }}^{-1} \sum_{p=1}^{N_{\text {config }}} T_{p}$ or, to have a more synthetic point of view, an average over the frequency range $0<\tilde{k}<1$, 


$$
\bar{T}=\frac{2}{P} \sum_{p=1}^{P / 2} T\left(k=\frac{2 \pi p}{L}\right)
$$

where the value $p \leq P / 2$ introduced in Section II.B. corresponds to $\tilde{k} \leq 1$. It is clear that $\overline{\langle T\rangle}=\langle\bar{T}\rangle$. Even if in a sense Eq. (8) seems to cover up information, this corresponds to a more realistic case, for which the source is not harmonic.

Even if this transmission coefficient is strongly frequency dependent, a first result is presented here at frequency $\tilde{k}=0.5$, in order to see how $\langle T\rangle$ in the middle of the transparency window is impacted by strong variation of couple $(a, L)$. For each couple of parameters $(a, L)$, the quantities $\left|p_{T}\right|^{2}$ and $\langle T\rangle$ are presented in Figure 4. On each curve presented in the two lower subplots in this Figure, each dot represents a transmission coefficient $T$ calculated for a given couple. For the largest radii $(a \geq 0.06 \lambda)$, a maximum appears after which $\langle T\rangle$ is exponentially decreasing. In order to enhance the analysis, simulations under the Born approximation (without particles interactions) are displayed on figures 4(b) and (d). In this case, the quantity $\left|p_{T}\right|^{2}$ never stops increasing with medium size $L$, showing the cumulative effect of the simple scattering in the direction of propagation of the source. It also increases with particles size $a$, because the total scattering cross section is increasing with $a$ at a given wavelength.

Having all that in mind, one can deduce from cartographies in Figure 5 what is the limit for transmission performances considering a given particles size and a given medium size. The four examples of $\langle T\rangle$ with respect to the frequency plotted in the same figure also show that the evolution of $\langle T\rangle$ with frequency can be very complicated when increasing $a$, even if an average process is performed over 20 different SHU configurations. This justifies the introduction of average transmission coefficient $\overline{\langle T\rangle}$ to study the transparency in a global manner.

As an important result of our study, we also connect decrease of transparency to the emergence of an incoherent part in the acoustic field. This incoherent part is visible on the cartography of $\delta(\overline{<T\rangle})=\overline{\left\langle(T-<T>)^{2}\right\rangle} / \overline{<T\rangle}$ in Figure 5(b). This quantity is the normalized standard deviation to the average field. For a SHU medium composed by point scatterers $(a \ll \lambda)$, it has to be 0 , because the scattered field is totally deduced from the structure factor, which is equally zero for all the disorder configurations. In other words, for this case, $<T>=T_{p}, \forall p$. When strong multiple scattering effects appear due to particles size or long propagation distance, an incoherent part appears $\left(<T>\neq T_{p}\right)$. From Figure 5 , we see a very good agreement between decrease of $\overline{\langle T\rangle}$ and increase of $\delta(\overline{\langle T\rangle})$.

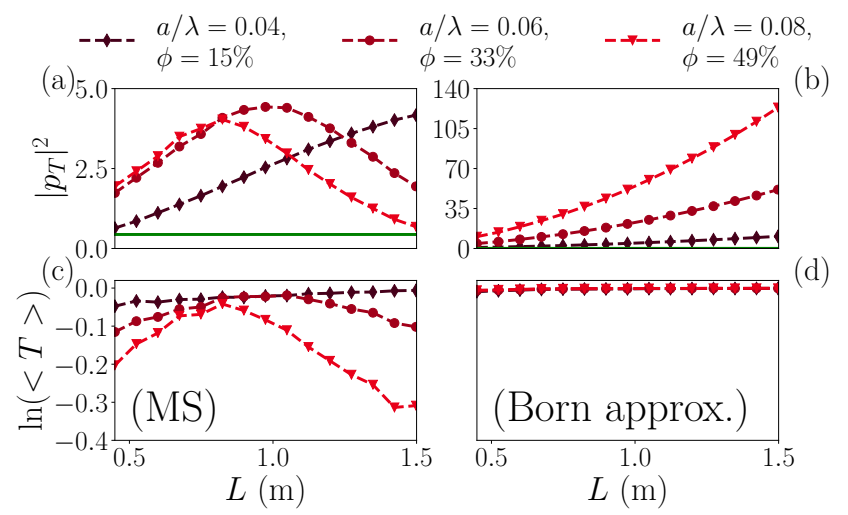

FIG. 4. (Color online) Scattered energy $\left|p_{T}\right|^{2}$ and transmission coefficient $T$ for different size of medium $L$ and different particles radii $a$, for $\tilde{k}=0.5$. Left panel shows results for Multiple Scattering simulation (MS) and right panel shows single scattering simulation (Born approximantion). The continuous (green) line represents the highest level of the reflection coefficient $R$. Every curve is calculated with MuScat and the point density $N / L^{2}=1200$ is kept constant.

If we compile these results, we first observe that transparency is more and more affected when the size of particles and the size of the medium increase, i.e. when the multiple scattering is reinforced and the distance on which waves propagate increases. In the latter case, there is a "conflict of interest" between the size of the medium and the elastic mean free path that is the distance on which the field is still ballistic. This last point is discussed in terms of effective attenuation in the next section.

\section{EFFECTIVE ATTENUATION IN SHU DENSE MEDIA WITH LARGE PARTICLES}

Now that transmission performances have been shown to strongly vary with parameters $(a, L, \lambda)$, we explore the question of the losses inside the medium. To do that, the effective attenuation is computed numerically and compared to different models from the literature.

\section{A. Criterion for transparency at large wavelength regime in finite medium}

Let us recall the effective wavenumber of the Independant Scattering Approximation (ISA) $k_{\text {ISA }}[11,22]$, also called Boltzmann approximation [30] that takes into account single scattering events, given by

$$
k_{\mathrm{ISA}}^{2}=k^{2}-4 i n_{0} f(0)
$$



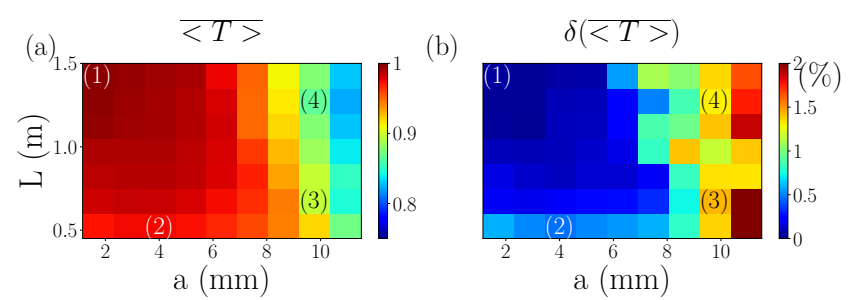

(1)

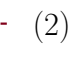

(3)

$(4)$

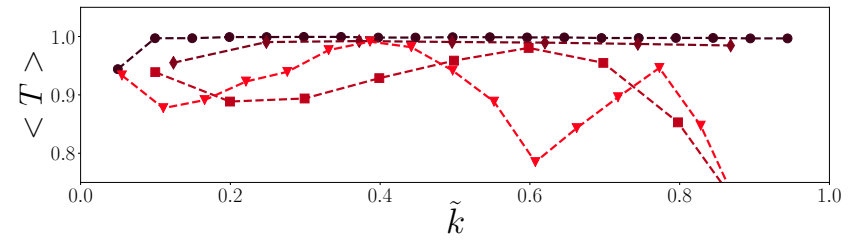

FIG. 5. (Color online) Mean of transmission coefficient $\overline{\langle T\rangle}$ over the frequency range $\tilde{k} \in[0,1]$, for different medium size $L$ and particles size $a(\mathrm{a})$. Standard deviation $\delta(\overline{\langle T\rangle})$ ) is presented (b), as well as four examples of transmission coefficients $\langle T\rangle$ for four couples of parameter $(a, L)$ with respect to the frequency (below). Number of cylinders is fixed such that $N / L^{2}=1200$.

where $f(\theta)=\sum_{n=-\infty}^{+\infty} T_{n} e^{i n \theta}$ is the far field form function, $n_{0}=\phi L^{2} / \pi a^{2}$ and $T_{n}$ the scattering coefficients of the particles. The scattering cross-section $\gamma_{L}$ is defined with the help of these coefficients as

$$
\gamma_{L}=\frac{2}{k a} \sum_{n=-\infty}^{+\infty}\left|T_{n}\right|^{2} .
$$

The ISA model, which is of first order in concentration, is not adapted to take into account geometrical constraints but considers the medium as purely uncorrelated. The corresponding scattering mean free path is given by $l_{e, \mathrm{ISA}}=1 /\left(2 \Im\left(k_{\mathrm{ISA}}\right)\right)$.

The question of evaluating the scattering mean free path $l_{e}$ in SHU media is not new. Leseur et al. used a diagrammatic approach based on the use of the selfenergy to derive the following order of magnitude (see Section 4 of Ref. [30])

$$
l_{e} \approx k l_{e, \mathrm{ISA}}^{2}
$$

in the frequency range $\tilde{k}<1$. It is noteworthy that Eq. (11) is only applicable for $\tilde{k}<1$. Furthermore, it is derived in under long-wavelength and independent scattering approximations. Considering the ballistic regime to be valid for $L<l_{e}$, with $l_{e}$ the real scattering mean free path of the SHU medium, they state that

$$
L<k l_{e, \text { ISA }}^{2}
$$

is the criterion for staying in the ballistic regime and ensuring transparency. The first question is whether the relation (11) is still correct for large particles ? Recent experimental work of Aubry et al. [26] showed that transparency seems to be conserved up to $\tilde{k} \rightarrow 1$, even for their dense system made of high index large particles for which relation (12) was not satisfied. Reacting to these results, we have investigated this problem in more details.

In the following, MuScat effective attenuation $\alpha_{\text {eff }}$, elastic mean free path $l_{e}$ and phase velocity $c_{\text {eff }}$ (useful for section V.A.) are calculated numerically. The medium being located between $x=0$ and $x=L$, one has two different pressure fields $p_{0}$ and $p_{1}[22]$

$$
\begin{cases}p_{0}=A e^{i k L} & \text { in homogeneous medium, } \\ p_{1}=A e^{i k_{\mathrm{eff}} L} & \text { in heterogeneous medium }\end{cases}
$$

with $k_{\text {eff }}=\omega / c_{\text {eff }}+i \alpha_{\text {eff. }}$ Effective coefficients $\alpha_{\text {eff }}=$ $1 / 2 l_{e}$ and $c_{\text {eff }}$ are calculated using the expression

$$
\left\{\begin{array}{l}
\alpha_{\mathrm{eff}}=-\frac{1}{L} \ln \left(\left|\frac{p_{1}}{p_{0}}\right|\right) \\
c_{\mathrm{eff}}=\omega L\left[\frac{\omega L}{c_{0}}+\arg \left(\frac{p_{1}}{p_{0}}\right)\right]^{-1} .
\end{array}\right.
$$

\section{B. Effective attenuation in thin SHU materials with large particles at low concentration}

We focus now on the effective attenuation for SHU materials built with different values of $\chi$ and different concentrations $\phi$.

In this part, $K a / 2$ stays below $0.4(a=2.5 \mathrm{~mm})$. The size $L$ of the medium is chosen such that Eq. (12) is satisfied. Imposing these parameters, we focus on the stealthiness of the medium $\chi$ and its impact on the effective attenuation. In Figure 6, MuScat simulations as well as Leseur predictions [30] clearly indicate that attenuation stays very low compared to ISA for $\tilde{k}<1$ (the vertical dotted lines being the limit $\tilde{k}=1$ for each value of $\chi)$. It is worth noting that, for $\phi=10 \%$ and $\chi=0.55$, Leseur and MuScat predictions are in good qualitative agreement up to $k a>0.38$ for which attenuation starts increasing significantly. Very similar results are presented by Kim and Torquato for electromagnetic waves in Ref. [21], Our results can be seen as a numerical confirmation regardless the size of the scatterers of the analytical formulation developed by these authors. The original and key point of their model is to take into account $n$-point correlation functions of order higher than two for the effective dielectric tensor of electromagnetic waves propagating in random media. That way, they approximately accounts for spatial correlations at all orders between scatterers via the spectral density that appears in their so-called "attenuation function". In fact, the good agreement between the theory of Ref. [21] and the 


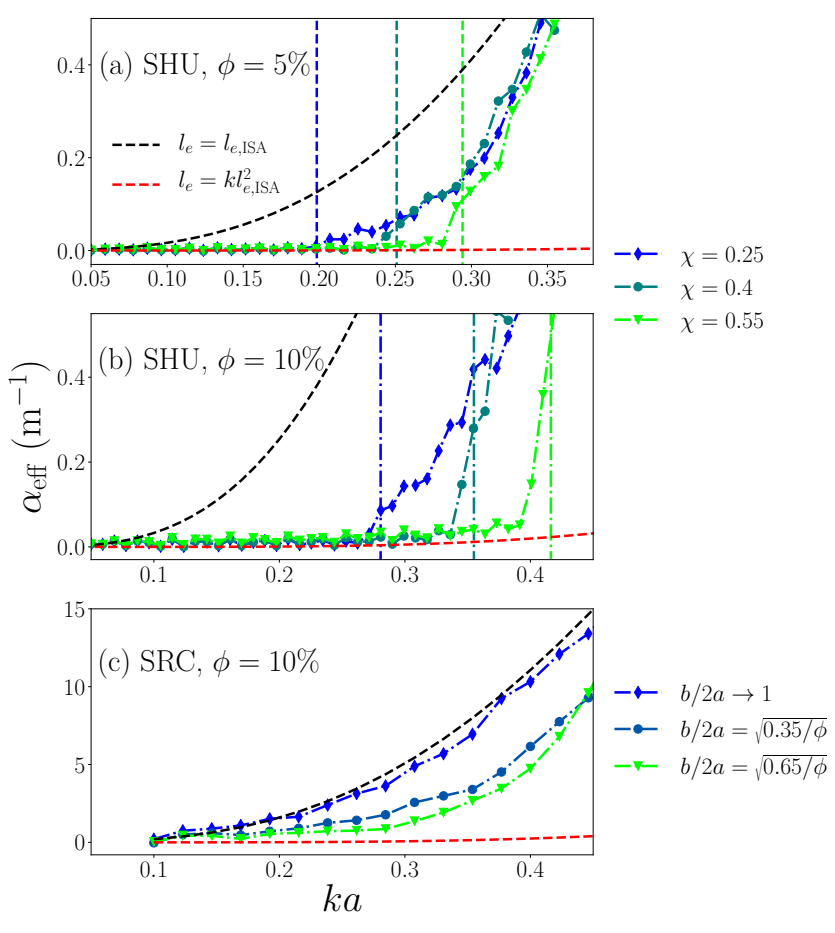

FIG. 6. (Color online) Effective attenuation for a medium with concentration $\phi=5 \%\left(N=1908, N / L^{2}=2550\right)$ (a) and $\phi=10 \%\left(N=3816, N / L^{2}=5100\right)(\mathrm{b}), a=2.5 \mathrm{~mm}$ and different values of parameter $\chi$. The vertical dotted lines correspond to the frequency for which $\tilde{k}=1$. (c) presents effects of SRC and is of the same type than results from [22], Figure 8(a).

results of the present paper shows that this theory correctly captures multiple scattering effects. In their work, they point out numerous applications. In particular, this shows that isotropic lowpassfilters can be built by introducing correlation into a purely random heterogeneous medium. To illustrate the link between SRC and SHU, we also present results similar to those of our earlier work [22] which focused on the impact of the increase of SRC parameter $b$ on the propagation. Results in Figure 6(c) demonstrates that at low frequency the introduction of SRC also makes the medium more transparent but in a smoother way compared to SHU. From these results, we demonstrate that wavelength approximation of Eq. (11) seems well appropriate to this case for which concentration is low but with large particles and strong multiple scattering.

\section{Effective attenuation in thin SHU materials with large particles at high concentration}

To go further into this investigation, we discuss now about how the behavior of SHU materials changes when the particles size increases at constant wavelength. To achieve this, 20 point patterns are built with stealthiness $\chi=0.6$. Imposing $\phi \leq 50 \%$, Eq. (6) imposes $(k a)_{\max }=0.97$. Furthermore, we impose $a_{\max }=11.5$ $\mathrm{mm}$. For each point pattern, we performed simulations increasing particles radii, bearing in mind that multiple scattering effects become stronger. Results in Figure 7 show the evolution of the parameter $\alpha_{\text {eff. Four different }}$ wavelengths $\tilde{k}=0.5, \tilde{k}=0.75, \tilde{k}=0.9$ and $\tilde{k}=1.1$ have been chosen in order to have a large overview on what happens when increasing the ratio $a / \lambda$. For $a=$ $a_{\max }$, values of $\tilde{k}$ leads respectively to $(k a)_{\max }=0.48$, $(k a)_{\max }=0.72,(k a)_{\max }=0.864,(k a)_{\max }=1.067$.

For the lowest frequency ranges $\tilde{k}=0.5$ and $\tilde{k}=0.75$, Leseur predictions $\left(l_{e} \approx k l_{e, I S A}^{2}\right)$ and MuScat simulations quantitatively agree for all radii, proving the validity of Eq. (11) for large wavelength regime, even at very high concentration. In these cases, multiple scattering effects are weak enough to be quantitatively described by the ISA coupled with the long range correlation analysis.

The third case $\tilde{k}=0.9\left((k a)_{\max }=0.864\right)$ is still below the upper bound $\tilde{k}=1$. For this case, the concentration effects start playing an important role for $a=9.2 \mathrm{~mm}$ which gives $k a \approx 0.7$. This value is also in agreement with the extreme value $a=11.5 \mathrm{~mm}$ of case (b) and appears to be the limit of validity of the large-wavelength independent scattering regime of Eq. (11). For such high concentrations values, it is not surprising that the ISA analysis fails to describe properly the propagation. One should consider adding second order terms in the effective wavenumber.

This is also visible on the last case $(\tilde{k}=1.1)$, for which MuScat simulations and ISA predictions are in almost perfect agreement for $a \leq 9.2 \mathrm{~mm}$. After this value, the multiple scattering effects are too strong to be well approximated by the ISA.

This analysis leads to the conclusion that the model proposed by Leseur [30] is in quantitative agreement with MuScat simulations up to a ratio $k a \approx 0.7$. This limit appears to be related to the independent scattering approximation. Hence, we proved that this order of magnitude is particularly appropriate to evaluate the effective attenuation of a SHU material up to critical values $\phi=30 \%$ and $k a \approx 0.7$. MuScat simulations presented here have been performed for a medium size of $L=0.5 \mathrm{~m}$. Other values have been tested that gave the same results.

\section{MEDIA WITH VARIOUS PARTICLES SIZES: EXCEEDING THE LIMITS OF POINT PATTERNS ANALYSIS}

In this part, the purpose is to complete the analysis of the transparency and enrich the structure factor information by considering new characteristics of the particle as the polydispersity, particles with subwavelength resonances, and by inserting the SHU material in a dispersive random medium. 
$\phi(\%)$

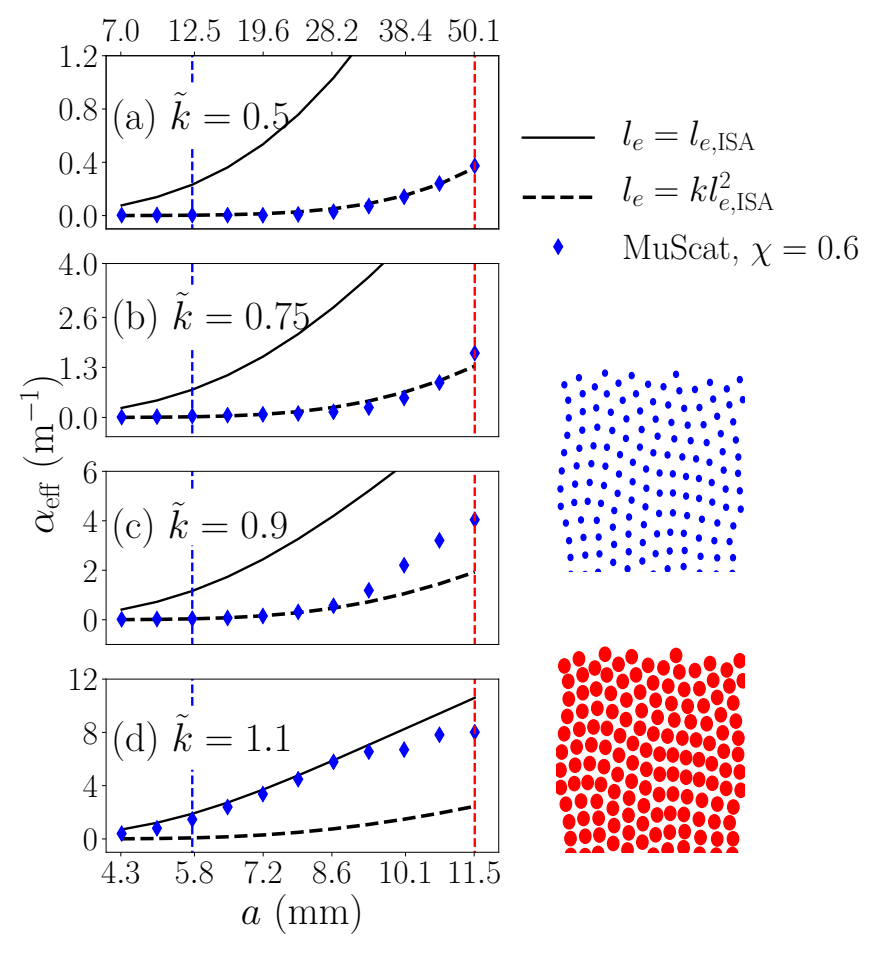

FIG. 7. (Color online) Effective attenuation at four wavenumber $\tilde{k}=0.5$ (a), $\tilde{k}=0.75$ (b), $\tilde{k}=0.9$ (c) and $\tilde{k}=1.1$ (d) as a function of particles size for a SHU medium with parameter $\chi=0.6$ and $N / L^{2}=600$. Vertical dotted lines indicate the radii used to build distributions on the right, which are illustrative examples of SHU distributions smaller but similar to the ones used to perform the simulation with MuScat.

\section{A. Heterogenous host medium}

What happens if the host medium that surrounds the large particles building the SHU material is heterogeneous? This question has already been discussed by Kim and Torquato [37] for point particles. They show that increasing the concentration of defects implies the loss of hyperuniformity. Here, to study this problem staying within the framework of multiple scattering, the host medium is made heterogeneous and dispersive by inserting small particles randomly distributed around the large ones (see Figure 8). More specifically, we built a SHU point pattern with $\chi=0.6, N=2700$ and $a=3.8$ mm. Then, we randomly added $N_{s}$ small particles inside this medium. To ensure that our study remains in the framework of SHU materials, we assume that multiple scattering effects associated to the host random medium are weaker than those related to the SHU material. This lead us to impose two limits, namely, the radius of the $N_{s}$ particles is equal to $a / 2$ (in order to reduce their scattering cross sections, the ratio of the $T_{0}$ scattering coef- ficients being approximately of 0.25 for the whole frequency range) and $N_{s} \leq 4 N$ so that the concentration $\phi^{\prime}$ of small particles stays below the concentration $\phi$ of the SHU material (both concentrations are equal when Ns $=4 \mathrm{~N}$ ). Before going further, using the numerical procedure described in previous part, the effective wavenumber $k_{\text {eff }}=\omega / c_{\text {eff }}+i \alpha_{\text {eff }}$ that characterizes the heterogeneous host medium is shown in Figure 9. As expected at low frequencies, one can note that the dispersion of the velocity is rather weak but the attenuation is increasing.

In order to study the propagation through the medium, the transmission coefficient $\tilde{T}$ is introduced as follows

$$
\tilde{T}=\frac{\left|p_{T}\right|^{2}}{\left|p_{R, h}\right|^{2}+\left|p_{T, h}\right|^{2}} \approx \frac{\left|p_{T}\right|^{2}}{\left|p_{T, h}\right|^{2}}
$$

where $p_{R, h}$ and $p_{T, h}$ are respectively the back-scattered and forward-scattered pressure for the case $N_{s}=0$, consisting of a purely homogeneous host medium. The quantity $\left|p_{R, h}\right|^{2}$ is small compared to $\left|p_{T, h}\right|^{2}$. This allows us to write approximation of Eq. (15). $T$ and $\tilde{T}$ give different types of information. As previously, $T$ gives the ratio between reflection and transmission. On the other hand $\tilde{T}$ allows to estimate the losses due to the dispersion for different degrees of short-scale heterogeneity (number of small particles $N_{s}$ ) in the host medium.

It is worth noting in Figure 8 that $\mathrm{T}$ remains close to unity and is significantly modified only for large values of $N_{s}$. Transmission performances stay very high, which endorses the fact that this phenomenon is resistant to the dispersion even if the transmitted field intensity, represented by $\tilde{T}$, undergoes a significant decrease.

One must keep in mind that results presented here are only valid if the two populations (namely the $N$ and the $N_{s}$ particles) can be studied separately. Here, we show that the whole medium can be understood as the superposition of a SHU material immersed in a dispersive medium. This unexpected result has a limit of course, especially in term of concentration, but this limit is out of the scope of this paper. However, this result is significant because it shows how a particles size study can reveal SHU properties where the simple point pattern analysis fails.

To support this result and in order to go further, we will now examine whether the SHU medium surrounded by the dispersive random medium preserves its main characteristic, namely the cancellation of the structure factor. From a first point of view, based on homogenization approaches, assuming the SHU immersed in the effective medium amounts to consider that the quantity $S(k)$ is computed taking $\mathbf{k}=\left(k_{x}, k_{y}\right)^{t}=\left(\frac{c}{c_{\text {eff }}} \frac{2 \pi}{L}+\right.$ $\left.i \alpha_{\text {eff }}\right)\left(n_{x}, n_{y}\right)^{t}$, with $-P / 2<\left(n_{x}, n_{y}\right)<P / 2$. This leads to the structure factors plotted in Figure 10 (b) (d), where $\phi$ stands for the concentration of the SHU 
$N$-points pattern and $\phi^{\prime}$ for the effective host medium composed by the $N_{s}$ particles. The structure factor almost perfectly cancels out and the transmission behavior can be explained in simple terms, as a direct consequence of the vanishing of the structure factor which depends on the effective wavenumber.

To explore the subject further, it is also interesting to examine what happens when considering the whole point pattern in the structure factor calculation, taking into account the particles size through the scattering coefficient $T_{0}$, in the manner of Romero-García et al. [38]

$$
S\left(\mathbf{k}, T_{0}\right)=\frac{1}{\sum_{j=1}^{N+N_{s}} T_{0}^{(j) 2}} \sum_{j=1}^{N+N_{s}} \sum_{k=1}^{N+N_{s}} T_{0}^{(j)} T_{0}^{(k)} e^{i \mathbf{k} \cdot\left(\mathbf{r}_{\mathbf{k}}-\mathbf{r}_{\mathbf{j}}\right)},
$$

with the idea that taking into account the type of particles gives a different weight for the large compared to the small ones (note that Eq. (16) is equivalent to Eq. (2) if all the scatterers are identical). We chose here to keep only the monopolar mode because the definition was derived for point scatterers [38]. The resulting structure factor is plotted for two values of $N_{s}$ in Figures 10 (a),(c). We clearly see in these Figures that the domain $\Omega$ is identifiable, even if the structure factor is not equal to zero. However, the structure factor appears to be affected homogeneously in the entire domain $\Omega$, which is in less quantitative agreement with the observed weakening of the transmission coefficient $T$ that is most affected for $\tilde{k}$ close to 1 .

As strong and important result of this study, we show that a complex medium made of two distinct populations of different sizes can be successfully understood as the superposition of a dispersive random medium (that can be homogenized) and a SHU medium that keeps its transparency properties. These properties are only affected by the dispersion of the host medium, if the concentration stays rather low.

\section{B. Influence of polydispercity}

This part deals with the question of the influence of polydispersity. Nature never reaches perfect monodispercity, and laboratory conditions for experiment also integrates error bars on the size of the particles. The proof of the great importance of accounting for polydispercity in many cases is already established [39]. Here, the introduction of such effects is possible thanks to the $\mathbb{T}$-matrix formalism that allows to change one by one the properties of each particle. The mean radius is chosen to be $a_{0}=1$ $\mathrm{mm}$, and the variations are random, following a Gaussian distribution of standard deviation $\sigma$, with imposed upper limit $a_{\max }=2 a_{0}$ to ensure no interpenetrability between particles and lower limit $a_{\min }=1 \mu \mathrm{m}$. Here, parameter $\chi=0.5$. Figure 12 shows that transmission is

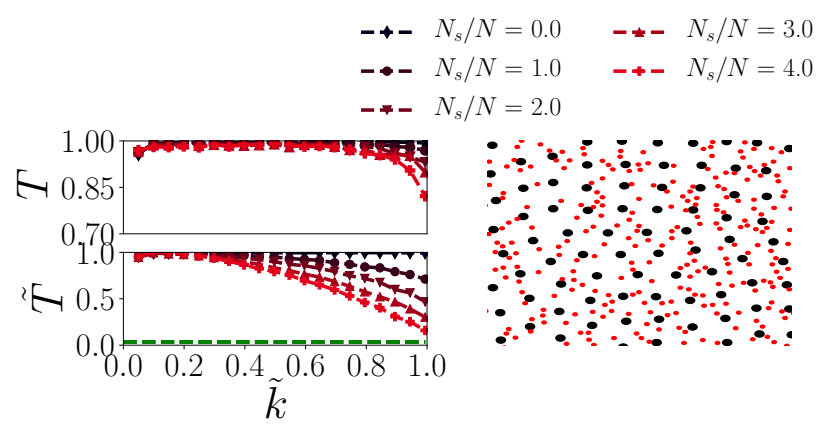

FIG. 8. (Color online) Transmission coefficient $T$ and $\tilde{T}$ for media composed of a stealthy distribution of $N$ particles with radius $a$ (black circles), $N / L^{2}=1200$ and a uncorrelated distribution of $N_{s}$ particles of radius $a / 2$ (red/light grey circles). (Green) dotted line represents the stronger level of reflection coefficient $\tilde{R}$, whose definition is analogous to $\tilde{T}$.
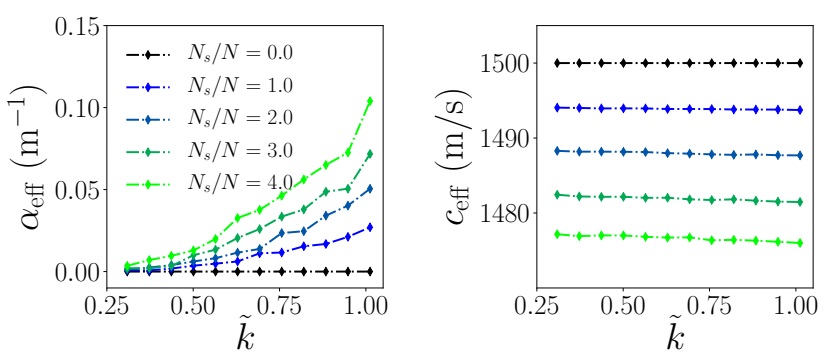

FIG. 9. (Color online) Effective parameters $\alpha_{\text {eff }}$ and $c_{\text {eff }}$ of the medium composed by the $N_{s}$ particles with respect to the dimensionless wavenumber $\tilde{k}, K$ being the one used in Figure 8. The case $N_{s}=0$ stands for the non dispersive homogeneous medium.

weakened by polydispersity. However, considering experimental uncertainty, for which $10 \%$ of variation is quite important, it is worth noting that transmission stays very close to 1 up to $\tilde{k}=1$.

Gathering this conclusion with previous ones, it is meaningful to remember that all the particles do not play the same role in the propagation inside the structure. With the use of Eq. (16), the structure factor is calculated for the case $\sigma=0.3 a_{0}$ and presented in Figure 11(b). Comparing with the monodisperse case (a), one can observe that the resulting structure is no more stealthy, mainly for the highest wavenumbers in the square, as shown in Figure 11(b). This is in agreement with the fact that $T$ starts decreasing before $\tilde{k}=1$. However, having in mind experimental constraints, it is worth noting that transmission stays very close to 1 for relatively large polydispersity. 
(a) $N_{s} / N=1$

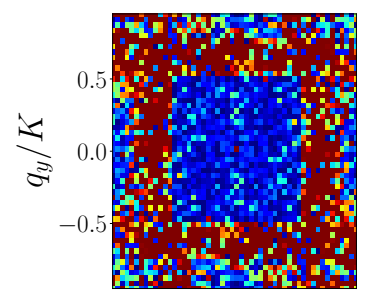

(c) $N_{s} / N=4$

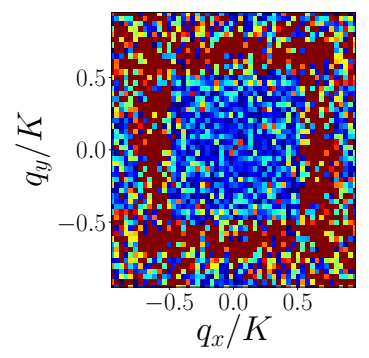

(b) $\phi^{\prime} / \phi=0.25$
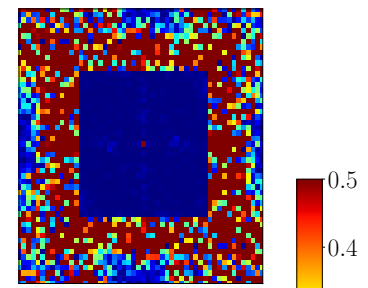

(d) $\phi^{\prime} / \phi=1$

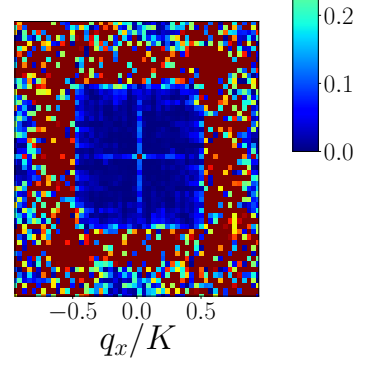

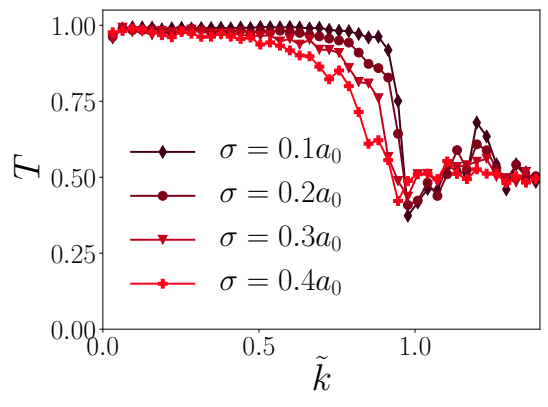

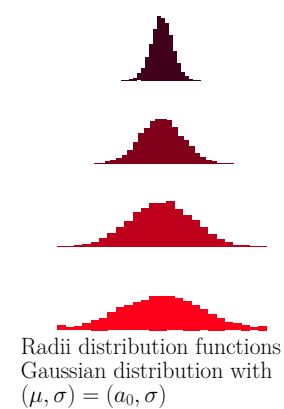

FIG. 12. (Color online) Transmission coefficient through polydisperse media with various radii distribution functions, $\chi=0.5$ and $N / L^{2}=26460$.

often a limiting factor and in this context, SHU appears as a promising candidate due to their transparency property. That leads then to the question of the influence of a resonance on the propagation of waves through a SHU material. To achieve this, we consider steel shells filled by water, with outer radius $a_{\text {out }}=5 \mathrm{~mm}$ and various inner radius $a_{\text {in }}$ that drive the resonance frequency of the mode of vibration $n=1$.

Figure 13 shows the impact of the resonance on the coefficient of transmission $T$, reducing it by a factor of 0.65 close to resonance frequencies. This effect can be enhanced, by using more complicated resonators, but this is out of the scope of our study. The important thing is not this point but the fact that resonances perturb the transparency of the SHU material. It is not easy to explain this phenomenon. One hypothesis is as follow; periodic and SHU media have in common to involve interference phenomena. Bragg interferences for periodic media and cancellation of the structure factor for SHU materials.

For periodic media, a resonance results into the opening of a localized pass-band inside stop-bands [41, 42]. In other words, transmission is created in a frequency range where total reflection should be observed. This can be understood by remembering that stop-bands are due to Bragg interferences. When there is a resonance, waves scattered by the particles undergo a phase shift which varies very rapidly on the whole width of the resonance [42]. Such a phase shift locally destroys the interference phenomena that were responsible of the stop-bands. This explains why a localized pass-band is opened just around the resonance frequencies. The phenomenon is similar for SHU material. If we think that transparency is due to interferences, transparency must also be perturbed by the phase shift which varies very rapidly around the same resonance frequencies. In this case we observe in Figure 13 that the transparency is perturbed on the whole width of the resonance. Periodic and SHU materials are opposite from each other. In one case the resonance creates transmission, in the other it creates reflection. This suggests that SHU materials, like periodic ones, could be used for the construction of frequency filters. 


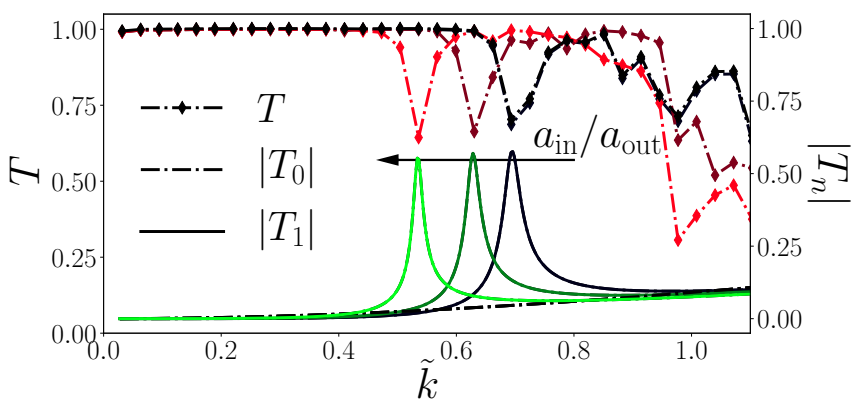

FIG. 13. (Color online) Transmission coefficient $T$ through resonant stealthy medium $\left(\chi=0.5\right.$ and $\left.N / L^{2}=26460\right)$ made of tubes of inner radius $a_{\text {in }}$ and outer radius $a_{\text {out }}$ for ratio $\frac{a_{\text {in }}}{a_{\text {out }}}=0.31,0.33,0.35$ (the arrow indicates the direction for which the ratio increases). The scattering coefficients $T_{0}$ and $T_{1}$ are also represented (right $y$-label, the resonance appears on the second scattering coefficient $T_{1}$ ).

\section{CONCLUSION}

This paper deals with the propagation of acoustic waves in two dimensional SHU medium made of elastic cylinders with large radii, with a particular focus on transparency. To achieve this, we have used the software MuScat [23] based on the modal decomposition of the fields that takes multiple scattering into account at all orders. In this context, we introduce an important acoustic parameter for realistic clustering distribution which is the concentration of particles. Once the position of cylinders is fixed, thanks to the degree of stealthiness $\chi[31]$, the concentration $\phi$ can be expanded by increasing the size of the cylinders. The perfect transmission property of SHU materials with non-ponctual particles has been demonstrated. However, it has been established that SHU materials are transparent only on condition that

$$
k a \leq \sqrt{\pi \phi \chi}
$$

Furthermore, it is observed that transmission performances strongly depend on three parameters: the radius of cylinders $a$, the size of the medium $L$ and the wavelength. In particular, it is shown that transparency disappears for higher values of $a$ and $L$, i.e. when the multiple scattering effect between particles increases with the onset of an incoherent field. From another analysis based on diagrammatic approach, Leseur et al. have proposed a order of magnitude for the scattering mean free path that depends on the scattering mean free path $l_{e, \text { ISA }}$ of uncorrelated random media [30]. We show that this analysis is still valid for large particles even for high concentrations but our investigation points out that the use of the independent scattering approximation in SHU must be put into question above and beyond $k a \approx 0.7$.

The question of the impact of polydispercity is also tackled and shows that, under common experimental uncertainties, transparency behavior is conserved. Further- more, it is shown that a SHU media also keeps its properties when immersed in an heterogeneous scattering background, in the limit of rather low concentration,

Lastly, by changing the elastic properties of our particles, we show how the introduction of a sub-wavelength resonance in the frequency range $\tilde{k}<1$ can be used as filter that stops the propagation of particular frequencies.

\section{ACKNOWLEDGMENTS}

We wish to thank Romain Pierrat (ESPCI ParisTech, PSL Research University, CNRS, Institut Langevin, Paris, France) for fruitful discussions.

\section{APPENDIX : CLUSTER EXPANSION IN TWO DIMENSIONS AND MUSCAT NUMERICAL PROCEDURE}

The total field resulting in the interaction of a source $p_{\text {inc }}$ and $N$ infinite cylinders located at $\left(\boldsymbol{r}_{1}, \boldsymbol{r}_{2}, \ldots, \boldsymbol{r}_{N}\right)$ in an homogeneous matrix can be written as

$$
p(\boldsymbol{r})=p_{\mathrm{inc}}(\boldsymbol{r})+\sum_{j=1}^{N} p_{s}^{(j)}\left(\boldsymbol{r}_{j}\right),
$$

where the acoustic pressure scattered by each cylinder is expanded on the cylindrical harmonics

$$
p_{s}^{(j)}\left(\boldsymbol{r}_{j}\right)=\sum_{n=-\infty}^{+\infty} A_{n}^{(j)} H_{n}^{(1)}\left(k r_{j}\right) e^{i n \theta_{j}}
$$

with $H_{n}^{(1)}$ the Hankel function of the first kind and order $n$ and $\left(r_{j}, \theta_{j}\right)$ the cylindrical coordinates of the point $\boldsymbol{r}$ in the coordinates system of the $j^{\text {th }}$ cylinder. For a given set of cylinders, the unknown amplitudes $A_{n}^{(j)}$ (truncated at order $N_{m}-1$ so that $2 N_{m}-1$ modes are taken into account) forming the vector $\mathbf{A}$ are determined by solving the following linear system

$$
[\mathcal{I}-\mathcal{T} \mathcal{M}] \mathbf{A}=\mathcal{T} \mathbf{E},
$$

with $\mathcal{T}$ the block matrix containing all the $\mathbb{T}^{(j)}$ (diagonal) matrices that contain the scattering coefficients of every scatterer $j$. The vector $\mathbf{E}$ is the modal amplitude of the incident wave and the matrix $\mathcal{M}$, built thanks to the Addition Theorem, models all the interactions between scatterers (neglecting it leads to the Born approximation).

The resolution of Eq. (19) is challenging for great numbers of cylinders or high frequencies. To achieve this, we use the in-house software MuScat [23] that is adapted for solving problems for sizes of several thousands of cylinders. It is worth pointing out that Eq. (19) allows the calculations of any kind of distributions without restriction concerning the number, the elastic properties, the 
source type nor the source frequency. A key point is that not only the monopolar mode is calculated here, but also the higher ones. In the following, $N_{m}-1$ will be the highest mode taken into account in the modal sum, and is computed for each case such as $\left|T_{\mathrm{N}_{\mathrm{m}}}\right|$ and $\left|T_{N_{m}+1}\right|<10^{-4}$.
[1] R. J. Elliot, J. A. Krumhans and P. L. Leath, Rev. Mod. Phys. 46, 465 (1974).

[2] A. Lagendijk and B. van Tiggelen, Phys. Rep. 270, 143 (1996).

[3] R. E. Challis, M. J. W. Povey, M. L. Mather and A. K. Holmes, Rep. Prog. Phys. 68, 1541 (2005).

[4] J. Mei, Z. Liu, W. Wen and P. Sheng, Phys. Rev. B 76, 134205 (2007).

[5] D. Torrent and J. Sánchez-Dehesa, Phy. Rev. B 74, 224305 (2006).

[6] E. Economou, Green's Functions in Quantum Physics, Vol. 1 (Springer-Verlag, 1979).

[7] L. Tsang and J. A. Kong, Scattering of Electromagnetic Waves: Advanced Topics (Wiley, 1995).

[8] P. Sheng, Introduction to Wave Scattering, Localization and Mesoscopic Phenomena (Academic, 1995).

[9] S. M. Rytov, Y. A. Kravtsov, and V. I. Tatarskii, Principles of Statistical Radiophysics, Vol. 4 (Springer-Verlag, 1989).

[10] L. Tsang and J.A. Kong and H. Habashy, J. Acoust. Soc. Am. 71, 552 (1982).

[11] A. Derode, V. Mamou and A. Tourin, Phys. Rev. E 74, 036606 (2006).

[12] M. Caleap, B. Drinkwater and P. D. Wilcox, J. Acoust. Soc. Am. 131, 2036 (2012).

[13] Andrew N Norris, Proceedings of the Royal Society A: Mathematical, Physical and Engineering Sciences 464, 2097 (2008).

[14] A. Gabrielli, M. Joyce, F. S. Labini, Phys. Rev. D 65, 083523 (2002).

[15] S. Torquato and F. H. Stillinger, Phys. Rev. E 68, 041113 (2003).

[16] Y. Jiao, T. Lau, H. Hatzikirou, M. Meyer-Hermann, J. C. Corbo, and S. Torquato, Phys. Rev. E 89, 022721 (2014).

[17] Y. Zheng, L. Liu, H. Nan, Z.-X. Shen, G. Zhang, D. Chen, L. He, W. Xu, M. Chen, Y. Jiao, and H. Zhuang, Science Advances 6 (2020).

[18] A. Donev, F. H. Stillinger, and S. Torquato, Phys. Rev. Lett. 95, 090604 (2005).

[19] J. Ricouvier, R. Pierrat, R. Carminati, P. Tabeling, and P. Yazhgur, Phys. Rev. Lett. 119, 208001 (2017).

[20] M. C. Rechtsman and S. Torquato, Journal of Applied Physics 103, 084901 (2008).

[21] J. Kim and S. Torquato, Proceedings of the National Academy of Sciences 117, 8764 (2020).

[22] A. Rohfritsch, J.-M. Conoir, T. Valier-Brasier, and R.
Marchiano, Phys. Rev. E 101, 023001 (2020).

[23] A. Rohfritsch, J-M. Conoir, R. Marchiano and T. ValierBrasier, J. Acoust. Soc. Am. 145, 3320 (2019).

[24] M. Florescu, S. Torquato, and P. J. Steinhardt, Proceedings of the National Academy of Sciences 106, 20658 (2009).

[25] L. S. Froufe-Pérez, M. Engel, P. F. Damasceno, N. Muller, J. Haberko, S. C. Glotzer, and F. Scheffold, Phys. Rev. Lett. 117, 053902 (2016).

[26] G. J. Aubry, L. S. Froufe-Pérez, U. Kuhl, O. Legrand, F. Scheffold, and F. Mortessagne, Phys. Rev. Lett. 125, 127402 (2020).

[27] W. Man, M. Florescu, E. P. Williamson, Y. He and S. R. Hashemizad, B. Y. C. Leung, D. R. Liner, S. Torquato, P. M. Chaikin, P. J. Steinhardt, Proceedings of the National Academy of Sciences 110, 15886-15891 (2013).

[28] G. Gkantzounis, T. Amoah, and M. Florescu, Phys. Rev. B 95, 094120 (2017).

[29] M. Florescu, P.J. Steinhardt and S. Torquato, Phys Rev B 87, 165116 (2013).

[30] O. Leseur, R. Pierrat, and R. Carminati, Optica 3, 763 (2016).

[31] S. Torquato, Physics Reports 745, 1 (2018).

[32] B. X. Wang and C. Y. Zhao, J. Opt. Soc. Am. B 37, 1757 (2020).

[33] O. U. Uche, F. H. Stillinger, and S. Torquato, Phys. Rev. E 70, 046122 (2004).

[34] J. Kim and S. Torquato, Phys. Rev. E 99, 052141 (2019).

[35] R. D. Batten, F. H. Stillinger and S. Torquato, J. Appl. Phys. 104, 033504 (2008).

[36] S. Torquato, Random Heterogeneous Materials: Microstructure and Macroscopic Properties (SpringerVerlag, New-York, 2002).

[37] J. Kim, and S. Torquato, Phys. Rev. B 97, 054105 (2018).

[38] V. Romero-Garcìa, N. Lamothe, G. Theocharis, O. Richoux, and L. M. Garcì-Raffi, Phys. Rev. Applied 11, 054076 (2019).

[39] R. Dreyfus, Y. Xu, T. Still, L. A. Hough, A. G. Yodh, and S. Torquato, Phys. Rev. E 91, 012302 (2015).

[40] M. L. Cowan, J. H. Page, and P. Sheng, Phys. Rev. B 84, 094305 (2011).

[41] M. Lanoy, J. H. Page, G. Lerosey, F. Lemoult, A. Tourin, and V. Leroy, Phys. Rev. B 96, 220201(R) (2017).

[42] J.-M. Conoir, J.-L. Izbicki, and O. Lenoir, Ultrasonics 35, 157 (1997). 